\title{
Le pingo d'Aishihik, sud-ouest du Yukon : caractères morphogénétiques et cadre temporel
}

The Morphology, Genesis and Temporal Context of the Aishihik Pingo (SW Yukon)

\section{Der Pingo von Aishihik, Süd-West Yukon: morphogenetischer Kontext und zeitlicher Rahmen}

\section{Marie-Anne Geurts et Véronique Dewez}

Volume 39, numéro 3, 1985

URI : https://id.erudit.org/iderudit/032609ar

DOI : https://doi.org/10.7202/032609ar

Aller au sommaire du numéro

\section{Éditeur(s)}

Les Presses de l'Université de Montréal

ISSN

0705-7199 (imprimé)

1492-143X (numérique)

Découvrir la revue

Citer cet article

Geurts, M.-A. \& Dewez, V. (1985). Le pingo d'Aishihik, sud-ouest du Yukon : caractères morphogénétiques et cadre temporel. Géographie physique et Quaternaire, 39(3), 291-298. https://doi.org/10.7202/032609ar
Résumé de l'article

Les nouvelles observations sur la structure externe et interne de la butte d'Aishihik confirment qu'on se trouve en présence d'un pingo, classiquement interprété comme étant de système fermé, alors qu'il se situe à la limite sud du pergélisol discontinu. Les éléments externes favorables à cette interprétation sont: la forme conique, impliquant un rapport hauteur/largeur $(4,75 / 44 \mathrm{~m}) \mathrm{de}$ l'ordre de $10 \%$, un cratère, ainsi qu'un milieu palustre et lacustre. À ceci s'ajoutent des éléments internes décelés par sondage : un noyau de glace pure de $193 \mathrm{~cm}$ d'épaisseur protégé par une couverture de sédiments meubles, la forme convexe des lentilles de glace pure constituées de monocristaux et la présence de matériel non gelé à la base. Des cassures avec et sans rejet témoignent également des pressions développées lors de la croissance du pingo. Des profils thermiques effectués dans la couverture indiquent que le pingo est en équilibre avec le climat actuel $\left(\mathrm{T}^{0}\right.$ moyenne annuelle de $\left.-4,4^{\circ} \mathrm{C}\right)$. Des indices palynologiques indiquent que les silts, qui constituent le flanc du pingo, ont été déposés depuis le tardiglaciaire. L'analyse minéralogique démontre que le pergélisol existait avant le dépôt de la White River Ash (1230 $\mathrm{BP})$. Quelques indices palynologiques suggèrent que le pingo était en croissance avant le dépôt de la White River Ash, et qu'il a repris son activité après cet événement.
Tous droits réservés @ Les Presses de l'Université de Montréal, 1985
Ce document est protégé par la loi sur le droit d'auteur. L'utilisation des services d’Érudit (y compris la reproduction) est assujettie à sa politique d'utilisation que vous pouvez consulter en ligne.

https://apropos.erudit.org/fr/usagers/politique-dutilisation/ 


\title{
LE PINGO D'AISHIHIK, SUD-OUEST DU YUKON: CARACTĖRES MORPHOGÉNÉTIQUES ET CADRE TEMPOREL
}

\author{
Marie-Anne GEURTS et Véronique DEWEZ, Département de géographie, Université d’Ottawa, 165, rue Waller, Ottawa, \\ Ontario K1N 6 N5.
}

RÉSUMÉ Les nouvelles observations sur la structure externe et interne de la butte d'Aishihik confirment qu'on se trouve en présence d'un pingo, classiquement interprété comme étant de système fermé, alors qu'il se situe à la limite sud du pergélisol discontinu. Les éléments externes favorables à cette interprétation sont: la forme conique, impliquant un rapport hauteur/largeur $(4,75 / 44 \mathrm{~m})$ de l'ordre de $10 \%$, un cratère, ainsi qu'un milieu palustre et lacustre. À ceci s'ajoutent des éléments internes décelés par sondage: un noyau de glace pure de $193 \mathrm{~cm}$ d'épaisseur protégé par une couverture de sédiments meubles, la forme convexe des lentilles de glace pure constituées de monocristaux et la présence de matériel non gelé à la base. Des cassures avec et sans rejet témoignent également des pressions développées lors de la croissance du pingo. Des profils thermiques effectués dans la couverture indiquent que le pingo est en équilibre avec le climat actuel ( $\mathrm{T}^{\circ}$ moyenne annuelle de $-4,4^{\circ} \mathrm{C}$ ). Des indices palynologiques indiquent que les silts, qui constituent le flanc du pingo, ont été déposés depuis le tardiglaciaire. L'analyse minéralogique démontre que le pergélisol existait avant le dépôt de la White River Ash (1230 BP). Quelques indices palynologiques suggèrent que le pingo était en croissance avant le dépôt de la White River Ash, et qu'il a repris son activité après cet événement.
ABSTRACT The morphology, genesis and temporal context of the Aishihik Pingo (SW Yukon). These new observations of the external and internal structures of the Aishihik mound confirm that it is a pingo, classically interpreted as being of the closed-system type, whereas it is at the southern limit of discontinuous permafrost. The external evidence supporting this conclusion is the conical form, with a height/diameter ratio of the order of $10 \%(4,75 / 44 \mathrm{~m})$, a central crater, and a marshy and lacustrine environment. In addition to this, the internal evidence from the borings includes: a pure ice core $193 \mathrm{~cm}$ thick, with a mantle of unconsolidated sediments, the convexity of the monocristalline ice lenses, and the presence of unfrozen material at the base. Normal faults and tensional fractures further bear witness to the development of internal pressure during growth of the pingo. Thermal profiles of the mantle show that the pingo is in equilibrium with the present climate (mean annual temperature of $-4,4^{\circ} \mathrm{C}$ ). $\mathrm{Pa}$ lynological analyses suggest that the silts which form the flanks of the pingo were deposited in the late glacial period. Mineralogical analyses show that the permafrost predates the White River Ash (1230 BP). Some palynological data suggest that the pingo was growing prior to the deposition of the White River Ash, and was reactivated after this event.
ZUSAMMENFASSUNG Der Pingo von Aishihik, Süd-West Yukon: morphogenetischer Kontext und zeitlicher Rahmen. Die neuen Beobachtungen über die externe und interne Struktur des Hügels von Aishihik bestätigen, daß es sich um einen Pingo handelt, herkömmlich als dem geschlossenen System zugehörig interpretiert, während er sich an der südlichen Grenze des unterbrochenen Dauerfrosts befindet. Die externen Elemente, die eine solche Interpretation begünstigen, sind: die konische Form, die eine HöheDurchmesser-Beziehung $(4,75 / 44 \mathrm{~m})$ von $10 \%$ beinhaltet, ein Krater sowie ein Sumpf- und Seemilieu. Dazu kommen noch interne Elemente, die durch Probebohrungen entdeckt wurden: ein Kern aus reinem Eis von 193 $\mathrm{cm}$ Dicke, der durch eine Schicht lockerer Sedimente geschützt ist, die konvexe Form der Linsen aus reinem, monokristallinem Eis und das Vorhandensein von nicht gefrorenem Material an der Basis. Brüche mit und ohne Verwurf bezeugen ebenfalls die Entwicklung des Drucks während des Anwachsens des Pingo. In der Deckschicht durch geführte thermische Profile weisen darauf hin, daß der Pingo mit dem gegenwärtigen Klima im Gleichgewicht ist (durchschnittliche Jahrestemperatur von $-4,4^{\circ} \mathrm{C}$ ). Palynologische Anhaltspunkte lassen erkennen, daß der Schlamm, aus dem die Flanke des Pingo besteht, in der Späteiszeit abgelagert wurde. Die mineralogische Analyse zeight, daß der Dauerfrost vor der Ablagerung der White River Ash existierte (1230 v.u.Z.). Einige palynologische Anhaltspunkte lassen vermuten, daß der Pingo vor der Ablagerung der White River Ash existierte (1230 v.u.Z.). Einige palynologische Anhaltspunkte lassen vermuten, daß der Pingo vor der Ablagerung der White River Ash im Anwachsen war, und daß er nach diesem Ereignis wieder aktiv wurde. 


\section{INTRODUCTION}

La première mention de l'existence d'un pingo de système fermé à Aishihik a été publiée par HUGHES (1969), qui le situe simplement à l'intérieur des limites de la glaciation de McConnell (Wisconsinien supérieur) et de celles du pergélisol discontinu (fig.1).

Notre travail vise à décrire les caractères externes et internes de la butte d'Aishihik afin de déterminer s'il s'agit réellement d'un pingo et s'il est en équilibre avec son milieu actuel. L'étude tente également de replacer la butte dans un cadre temporel à l'aide d'analyses minéralogiques et polliniques.

\section{LE MILIEU ACTUEL}

La butte est située dans une vallée d'environ $500 \mathrm{~m}$ de large, et encaissée d'environ $30 \mathrm{~m}$ dans les terrasses glaciolacustres et fluvioglaciaires qui s'étendent au nord du lac Aishihik (longitude: $130^{\circ} 28^{\prime} 18^{\prime \prime} \mathrm{O}$, latitude: $61^{\circ} 4^{\prime} 24^{\prime \prime} \mathrm{N}$, altitude: ca $940 \mathrm{~m}$ ). Cette vallée, qui correspond à l'ancien exutoire du lac Aishihik (O. L. Hughes, comm. écrite), possède un plancher essentiellement silteux et une pente très faible vers le nord $(0,04 \%)$, ce qui explique le mauvais drainage, les nombreuses mares et le milieu palustre riche en Cypéracées (fig.2).

\section{DESCRIPTION DES CARACTĖRES DE LA BUTTE}

Deux profils établis au clinomètre Suunto PM-5 (fig.3-4) livrent les dimensions de cette butte de forme ovale; longueur: $44 \mathrm{~m}$, largeur : $30 \mathrm{~m}$, hauteur au-dessus du niveau du marais: $4,75 \mathrm{~m}$. La butte se caractérise par la présence d'un cratère et de flancs en pente raide atteignant localement $26^{\circ}$ (fig.5), qui sont affectés de solifluxion. Les versants ouest, sud et est portent une végétation rase constituée de graminées, de coussins d'Artemisia (sp) et quelques Potentilla (sp). Le versant nord et le cratère sont colonisés par une saulaie dense de 2,5 à $3 \mathrm{~m}$ de hauteur.

Des profils thermiques du sol ont été effectués le 11 août 1984 le long des axes sud-nord et ouest-est de la butte, avec un téléthermomètre YSI Modèle 42 SC. IIs définissent l'épaisseur de la couche dégelée à cette date (fig. 3 et 4). L'épaisseur du mollisol est minimale sur le versant nord, à insolation réduite, à pente forte, et protégé par la saulaie. Le mollisol s'épaissit sur les versants ouest, est et sud pour atteindre ses profondeurs maximales au sommet de la butte et dans le cratère.

Deux sondages réalisés avec une tarière motorisée portative et des carottiers CRREL modifiés, d'un diamètre utile de 7,6 et $5,1 \mathrm{~cm}$ (VEILLETTE et NIXON, 1980), ont procuré des carottes destinées à la description macroscopique des natures,

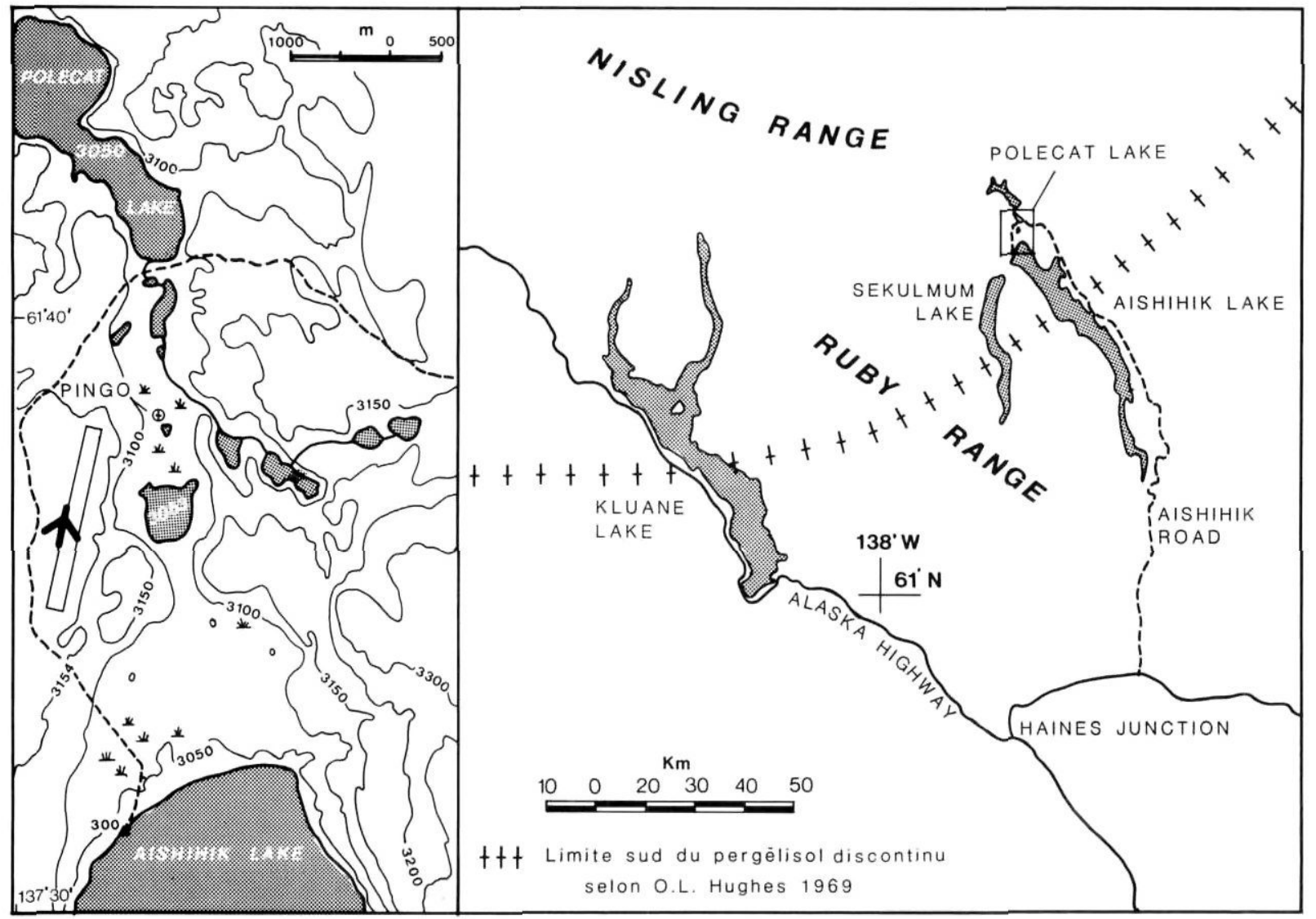

FIGURE 1. Carte de localisation. Le pingo est accessible par la route d'Aishihik et se trouve au N.E. du terrain d'aviation.
Location map. The pingo is to the north-east of the airfield, and is accessible via the Aishihik road. 


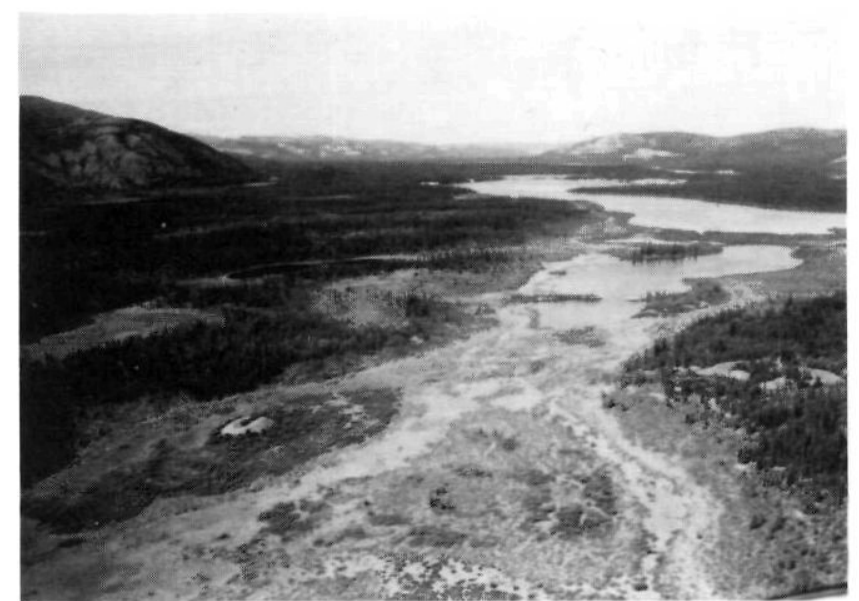

FIGURE 2. Vue en direction nord vers le lac Polecat. Le pingo est visible dans la partie inférieure gauche. Photographie: $\mathrm{H}$. Beaudet. View to north towards Polecat Lake. The pingo is at the bottom left. Photo: H. Beaudet. textures et structures des matériaux internes de la butte, et au prélèvement d'échantillons pour les analyses minéralogiques et polliniques.

Le sondage effectué au centre de la butte entre les $22^{\mathrm{e}}$ et $23^{e}$ stations du profil S-N a livré la stratigraphie suivante:

000-135 cm: sol dégelé et silteux avec horizon graveleux entre 80 et $100 \mathrm{~cm}$.

135-145 cm: sol gelé, silt et fines lentilles de glace de ségrégation disposées horizontalement.

145-147 cm: glace limpide en monocristaux.

147-162 cm: silt et lentilles de glace limpide de 1 à $1,5 \mathrm{~cm}$ d'épaisseur contenant des bulles.

162-355 cm: glace pure et limpide contenant des bulles, et se débitant en lentilles bombées vers le haut (convexes); leur épaisseur peut atteindre $4 \mathrm{~cm}$ (fig.6-7); les lentilles sont faiblement inclinées dans le carottier.

355-359 cm: silt gelé à glace cryptocristalline.
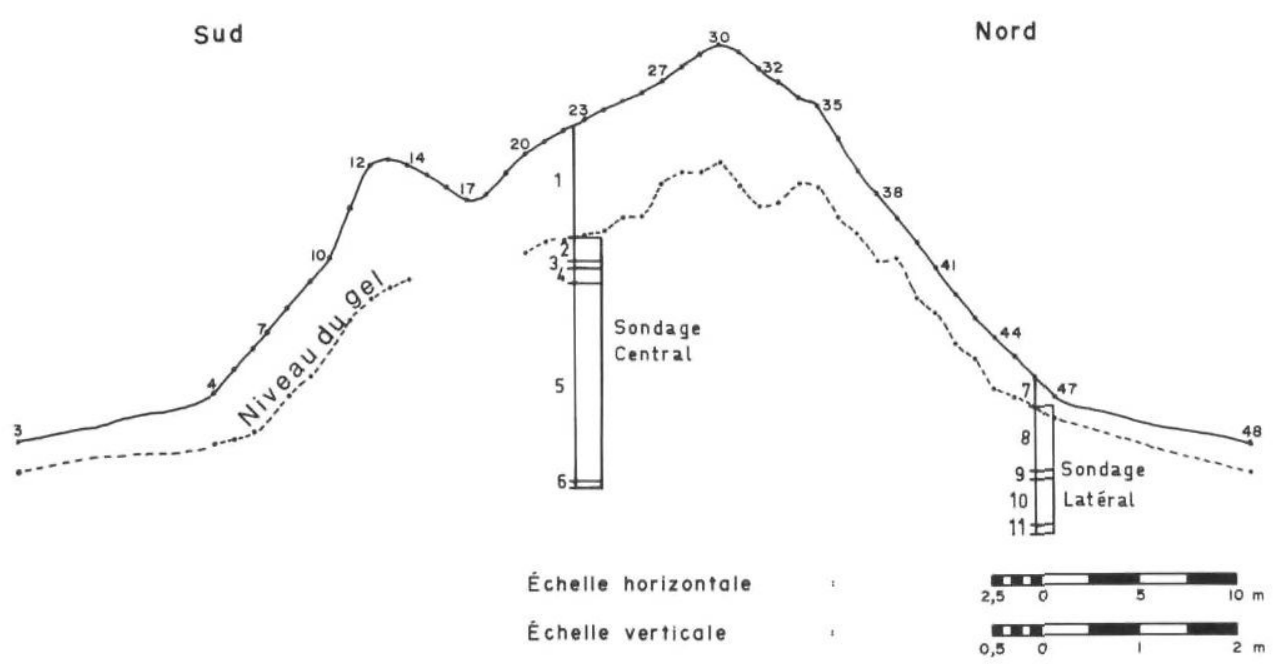

Exagération verticale 5
FIGURE 3. Profil topographique sud-nord du pingo et niveau du gel le 11 août 1984. Stratigraphie du sondage central: 1) sol silteux à horizon graveleux; 2) silt gelé à lentilles de glace de ségrégation; 3) glace limpide en monocristaux; 4) silt et lentilles de glace de ségrégation; 5) noyau de glace pure et limpide, contenant des bulles; 6) silt gelé à glace cryptocristalline. Stratigraphie du sondage latéral: 7) sol silteux riche en matière organique, cendre diffuse; 8) silt à lentilles de glace de ségrégation: 9) glace pure et limpide, contenant des bulles; 10) silt à lentilles de glace de ségrégation; 11) silt humide non gelé.

North-south topographical section of the pingo, showing the frost table on August 11 1984. Stratigraphy: see French text.

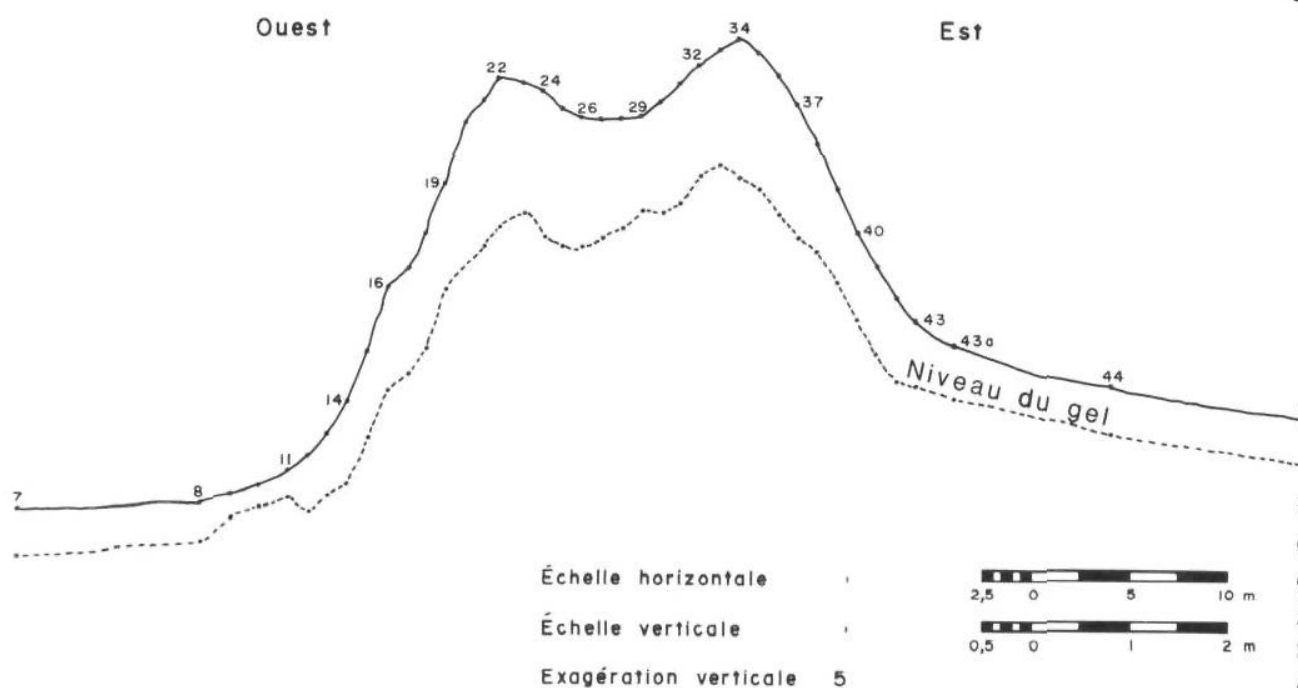

Exagération verticale 5
FIGURE 4. Profil topographique ouest-est du pingo et niveau du gel, le 11 août 1984.

East-west topographical section of the pingo, showing the frost table on August 11, 1984. 


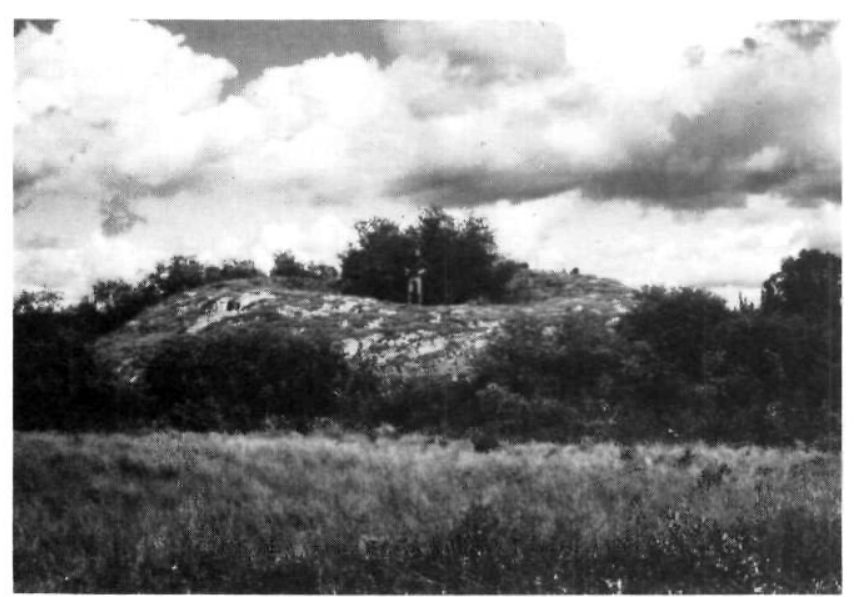

FIGURE 5. Vue sur les flancs nord et ouest du pingo. Photographie: M.-A. Geurts.

View of the north and west slopes of the pingo. Photo: M.-A. Geurts.

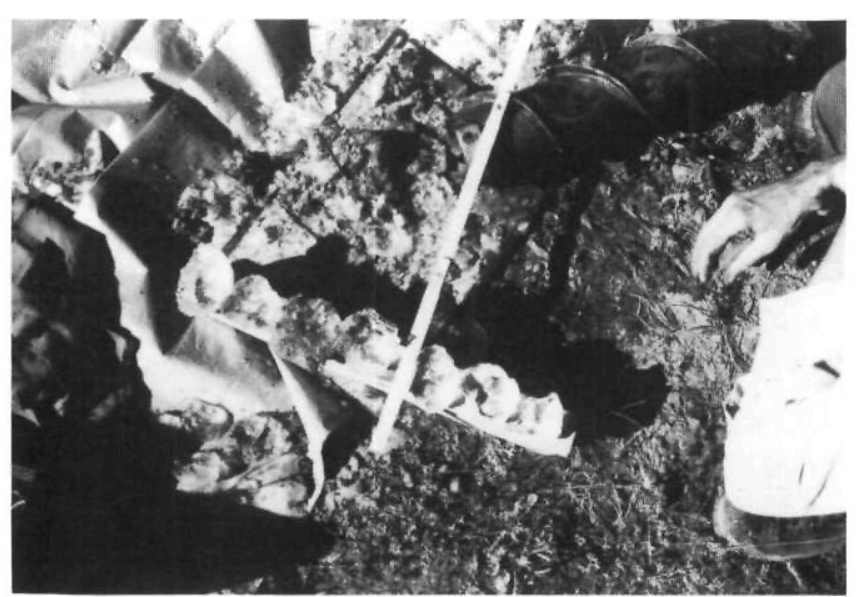

FIGURE 6. Lentilles convexes de glace pure, extraites par le carottier CRREL modifié, dans le sondage central. Photographie: G. Seret. Convex lenses of pure ice extracted by the modified CRREL corer, central hole. Photo: G. Seret.

La séquence décrite ci-dessus révèle la présence d'un noyau de glace pure d'au moins $193 \mathrm{~cm}$ d'épaisseur, et clivé en lentilles convexes. Cette structure particulière ne peut être imputée à la technique de sondage, puisque le carottier découpe par rotation une couronne de glace autour de la carotte elle-même (J. Veillette, comm. pers.). II s'agit donc bien d'une caractéristique inhérente au noyau de glace pure.

L'arrêt du sondage est d'ordre technique, et résulte de la présence à cette profondeur d'un matériel non pénétrable par la sonde. Ceci suggère la présence d'un horizon dégelé puisque la sonde ne fonctionne que dans le sol gelé.

Le deuxième sondage est localisé au niveau de la $45^{\mathrm{e}}$ station du transect S- $\mathrm{N}$, dans la saulaie, et a livré la séquence suivante:

000-008 cm: litière et horizon organique.

008-048 cm: horizon silto-organique s'enrichissant progressivement en argile; présence de la cendre de White River en faciès diffus entre 8 et $23 \mathrm{~cm}$; base du sol dégelé à $48 \mathrm{~cm}$.

048-103 cm: silt et lentilles de glace de ségrégation s'épaississant jusqu'à $8 \mathrm{~mm}$ (fig. 8 ); une faille subverticale est visible de 85 à $90 \mathrm{~cm}$; des cristaux de glace s'y sont développés perpendiculairement au plan de gel.

103-110 cm: glace pure et limpide, se débitant en deux grosses lentilles de 3 et $4 \mathrm{~cm}$ d'épaisseur; des bulles d'air sont visibles.

110-155 cm: silt et lentilles de glace de ségrégation (ca 8 $\mathrm{mm}$ ) et glace cryptocristalline vers $130 \mathrm{~cm}$. Une faille normale à rejet de quelques millimètres est visible vers $140 \mathrm{~cm}$ (fig.9).

155-165 cm: silt humide non gelé.

Le sondage a été arrêté en raison de l'impossibilité d'enfoncer le carottier dans l'horizon non gelé. Ceci confirme la présomption de la présence d'un horizon non gelé à la base du sondage central.
FIGURE 7. Disposition des lentilles de glace dans le carottier. Ice lenses as extracted in corer. 

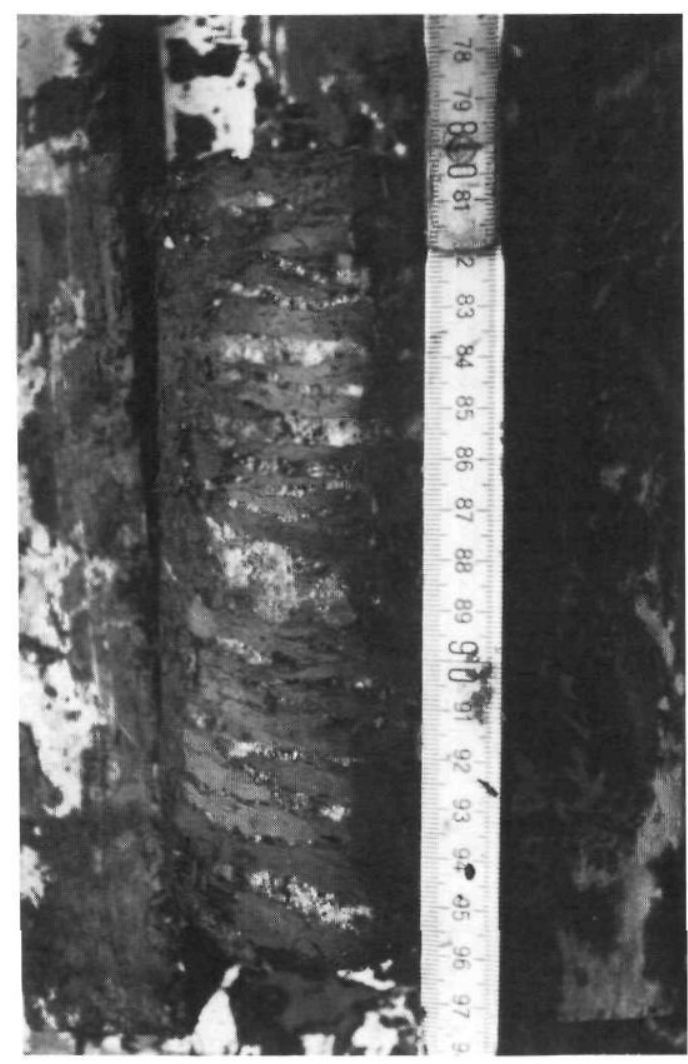

FIGURE 8. Silt et lentilles de glace de ségrégation entre 80 et 96 $\mathrm{cm}$ de profondeur dans le sondage latéral. Photographie: G. Seret. Silt and lenses of segregation ice from between 80 and $96 \mathrm{~cm}$ depth in the side hole. Photo: G. Seret.

\section{DISCUSSION}

Les descriptions ci-dessus livrent les éléments d'identification de la butte d'Aishihik.

1) La butte n'est certainement pas un frost blister (butte temporaire), tel que décrit par POLLARD et FRENCH (1983), puisqu'elle a déjà été mentionnée par HUGHES en 1969.

2) Cette butte n'est certainement pas une palse, qui selon la récente nomenclature de WASHBURN (1983), demeure une forme couverte de tourbe.

3) Elle ne présente pas les caractères d'une butte cryogène minérale (PISSART, 1983), puisque le noyau de glace pure est très épais et que les lentilles ne semblent pas constituées de glace de ségrégation. De plus, la butte est isolée.

4) La forme conique, le cratère, le rapport hauteur/longueur $(4,75 \mathrm{~m} / 44 \mathrm{~m})$ de l'ordre de $10 \%$ et la solifluxion sur les flancs suggèrent la comparaison avec les pingos tels que décrits dans la péninsule de Tuktoyaktuk (MACKAY, 1979).

5) Le sondage central, qui révèle un noyau de glace pure de $193 \mathrm{~cm}$ d'épaisseur, livre les éléments de comparaison les plus évidents. La forme convexe des lentilles de glace pure constituées de monocristaux indique une forte déformation interne et suggère une croissance centrale. Ceci relie la structure interne de la butte d'Aishihik au schéma théorique de

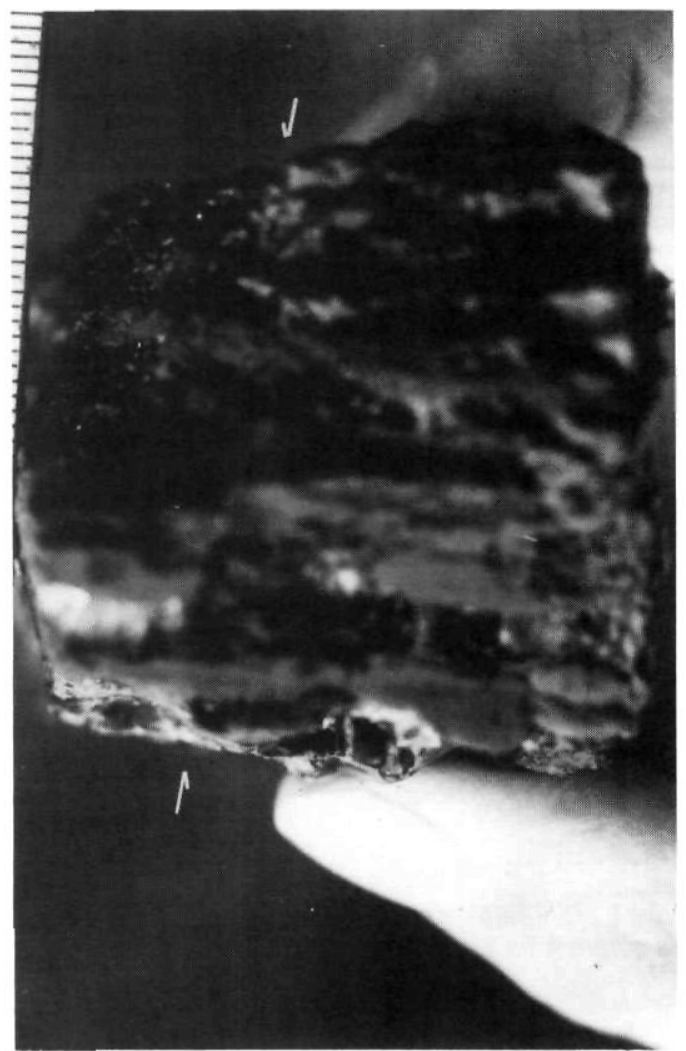

FIGURE 9. Faille normale à $140 \mathrm{~cm}$ de profondeur dans le sondage latéral. Photographie: G. Seret.

Normal fault at $140 \mathrm{~cm}$ in the side hole. Photo: G. Seret.

MACKAY (1979, fig.66A) des pingos à croissance centrale, qui selon cet auteur est le type de croissance le plus fréquent pour des pingos.

6) Dans le sondage latéral, deux grosses lentilles de glace pure ont été observées entre 103 et $110 \mathrm{~cm}$ de profondeur. Ces lentilles contiennent des vacuoles d'air comme la glace du sondage central. En dessous, on trouve du silt avec de la glace de ségrégation ou de la glace cryptocristalline. Cette succession évoque la bordure dentelée du noyau de glace du pingo $\mathrm{n}^{\circ} 9$ de la péninsule de Tuktoyaktuk (MACKAY, 1979, fig. 29).

7) La présence de matériel humide non gelé à la base du sondage latéral et l'impossibilité d'enfoncer davantage le carottier à la base du sondage central, qui suggère également du matériel non gelé, indique la présence du talik au moins latéralement sinon sous toute la surface du pingo.

8) Le noyau de glace situé entre 162 et $355 \mathrm{~cm}$ dans le sondage central est surmonté de $17 \mathrm{~cm}$ de grosses lentilles de monocristaux, à vacuoles d'air, interstratifiées au silt. Cette alternance de glace pure et de silt illustrerait les phases initiales de la formation du pingo.

9) Le sondage latéral a traversé une faille et une cassure ouverte. Ces cassures sont postérieures à l'engel strictement local et témoignent de pressions exercées lors de la croissance 


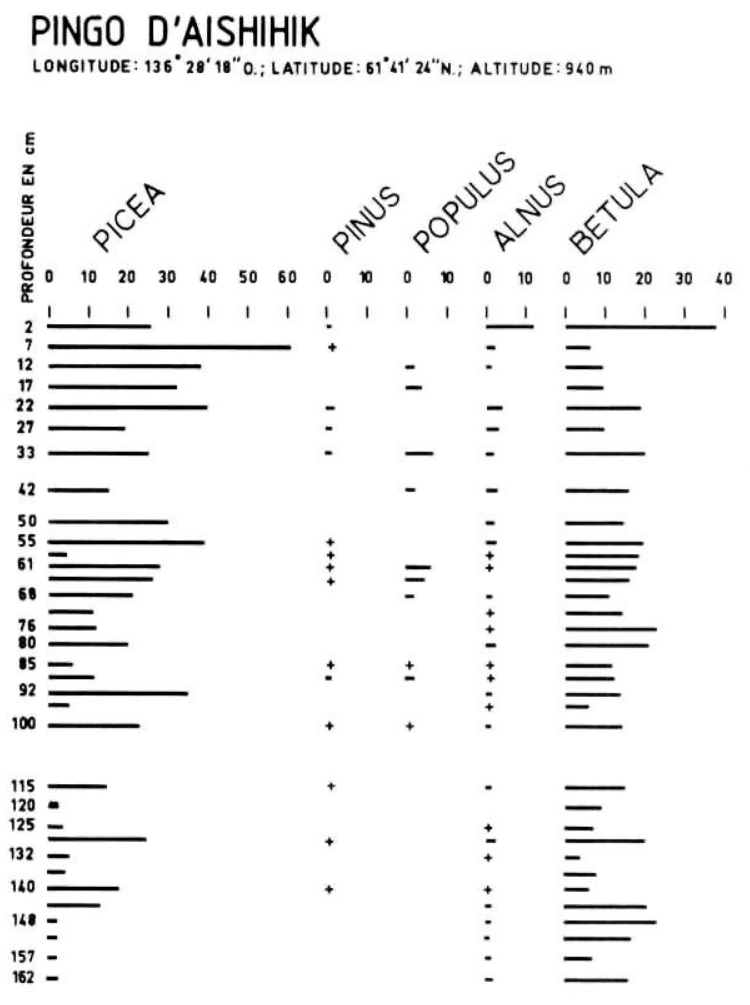

FIGURE 10. Diagramme pollinique du flanc nord du pingo. Pollen diagram from the north slope of the pingo.

du pingo, qui ont dépassé le seuil de cisaillement du matériel gelé. L'absence de fissures ouvertes en surface peut s'expliquer par l'efficacité de la solifluxion sur les versants.

10) Dans les deux sondages, la glace pure est surmontée de matériaux gelés, ce qui suggère que le noyau de glace reste actuellement à l'abri du dégel. Ces observations partielles sont corroborées par les profils thermiques qui situent le plan de dégel saisonnier bien au-dessus du noyau de glace. II apparaît donc que le pingo est en équilibre avec le climat actuel (station d'Aishihik: température annuelle moyenne de $-4,4^{\circ} \mathrm{C}$, Normales climatiques au Canada, 1982).

11) Comme l'a proposé HUGHES (1969), il s'agit fort probablement d'un pingo de système fermé en raison de l'environnement: une surface topographique plane, des versants peu importants en bordure de la vallée, ainsi que la présence de marais et mares résiduelles à proximité du pingo. De plus, cette vallée constitue l'ancien exutoire du lac Aishihik, et du silt a été observé dans les sondages du pingo et le long des berges du lac qui se trouve au nord du pingo. Une formation de silt glacio-lacustre de 15 m d'épaisseur s'observe en bordure du lac Aishihik. Elle est visible également sur les versants en arcs de cercle qui bordent l'entrée sud de l'ancien exutoire. L'exutoire du lac Aishihik s'est incisé dans ces formations, et le pingo posséderait donc un plancher silteux.

Dans ce cadre, le système fermé serait relié au caractère partiellement imperméable des matériaux sous-jacents, qui limiterait la vitesse de migration de l'eau dans le talik. Par conséquent, selon le concept de MACKAY (1979), lors de l'aggradation du pergélisol le système peut se fermer si l'expulsion de l'eau de pore (provenant des matériaux superficiels) excède la perte en eau en profondeur. II s'agirait donc d'un pingo de système hydrostatique.
L'ensemble de ces arguments confirment qu'il s'agit d'un pingo, à croissance centrale, en système fermé, qu'il est très probablement lié à des pressions hydrostatiques dues à l'aggradation du pergélisol, et enfin qu'il est en équilibre avec le climat actuel.

\section{CADRE TEMPOREL}

A défaut de matière organique datable au radiocarbone, l'étude tentera de reconstituer l'évolution du pingo en se basant sur les analyses minéralogiques, sur le cadre stratigraphique régional et sur les informations que l'on peut extraire des analyses polliniques.

\section{ANALYSES MINÉRALOGIQUES}

La cendre volcanique de "White River», dont le lobe est couvre la région d'Aishihik, est un repère lithostratigraphique daté en moyenne de 1230 BP (DENTON et KARLÉN, 1977). Celle-ci se caractérise par une abondance des hornblendes basaltiques (LERBEKMO et CAMPBELL, 1969).

L'analyse minéralogique des échantillons du sondage latéral montre que la White Rive Ash est bien présente, puisque les hornblendes basaltiques forment $79 \%$ du cortège des minéraux lourds (minéraux dont la densité dépasse 2,89, celle du bromoforme utilisé comme liqueur dense de séparation).

La proportion des hornblendes basaltiques diminue à environ $1 \%$ au sommet du pergélisol, et plus bas disparaissent du cortège minéralogique. Ceci signifie que le saupoudrage de la White River Ash a eu lieu après la formation du pergélisol, qui aurait empêché la diffusion plus profonde de la cendre par percolation ou solifluxion. Si le pergélisol se trouvait à même profondeur à 1230 BP qu'actuellement, il est fort possible également que le pingo existait avant $1230 \mathrm{BP}$. Les quelques 


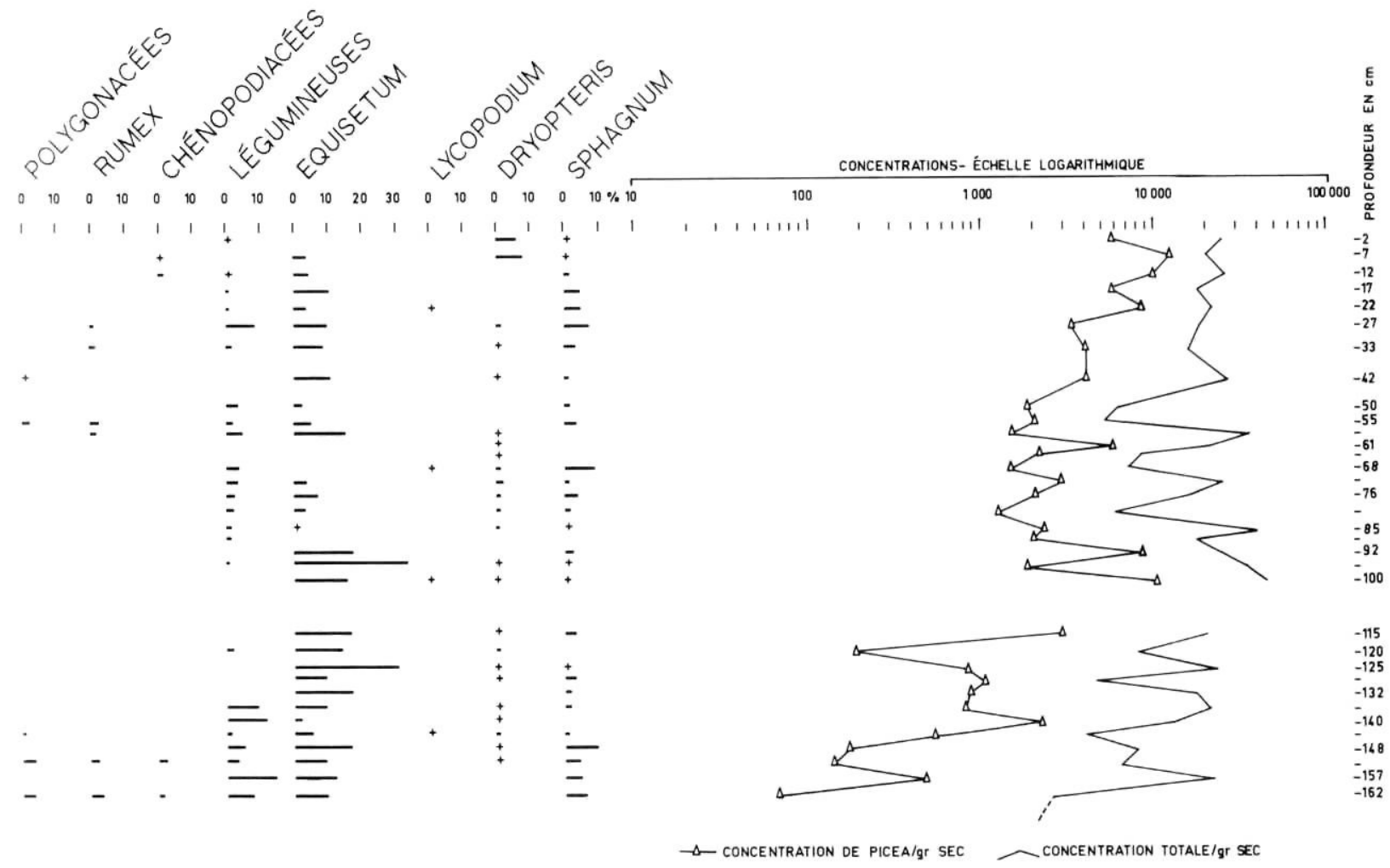

grains de hornblende basaltique observés au sommet du pergélisol s'expliquent par un battement vertical du plancher du mollisol en fonction des étés.

L'analyse minéralogique d'un échantillon de silt prélevé juste au-dessus du noyau de glace dans le sondage central ne révèle aucun grain de hornblende basaltique, ce qui confirme une croissance du pingo antérieure à l'éruption volcanique.

La cendre n'a pas été observée macroscopiquement dans les sédiments du sondage central. Son absence indiquerait que le cratère se serait ouvert après l'époque du saupoudrage. Le pingo aurait donc temporairement repris son activité après 1230 BP. L'absence d'accumulation de matière organique dans le cratère constitue un indice supplémentaire d'une ouverture relativement récente.

\section{CADRE STRATIGRAPHIQUE ET CHRONOLOGIQUE RÉGIONAL}

Une date de $7140 \pm 140$ BP (GSC-755) à la bordure actuelle du lac Aishihik donne l'âge minimal de la fin de l'épisode glaciolacustre de ce site (O. L. Hughes, comm. écrite). C'est donc au plus tard à cette époque que l'exutoire a pu être exposé à l'aggradation du pergélisol, si les conditions climatiques hivernales le permettaient.

\section{ANALYSES PALYNOLOGIQUES}

Le sondage latéral a permis de recueillir 34 échantillons entre 2 et $162 \mathrm{~cm}$ de profondeur. Tous les échantillons ont été traités par la méthode de Frenzel simplifiée par BASTIN (1971) et les résultats sont présentés en pourcentages calculés par rapport au total des AP (Arborean Pollen), ANP (Non Arborean Pollen) et des spores (fig.10). Le diagramme pollinique montre aussi les concentrations par gramme de la somme des grains de pollen et des spores, et celles des grains de pollen de Picea.
Le diagramme en pourcentage montre une augmentation progressive, mais avec de fortes fluctuations, de la fréquence de Picea depuis des valeurs inférieures à $5 \%$ jusqu'à un maximum de $61 \%$. Parallèlement à ceci, la concentration des grains de Picea passe de 70 grains $/ g$ à $162 \mathrm{~cm}$, à 12700 grains $/ \mathrm{g}$ à $7 \mathrm{~cm}$, et la concentration totale tend également à augmenter.

Comparativement à l'échantillon de $2 \mathrm{~cm}$ de profondeur (époque subactuelle), la concentration totale et la concentration de Picea sont importantes à $100 \mathrm{~cm}$ alors que la fréquence relative de ce pollen y est seulement de $22,5 \%$. On peut donc considérer qu'à l'époque représentée par l'échantillon de 100 $\mathrm{cm}$, les versants de la vallée étaient forestiers. Par conséquent, la sédimentation du silt s'est opérée pendant la période de croissance des apports de pollen de Picea dans le site, donc avant et pendant l'afforestation de la zone d'Aishihik.

Pour le sud ouest du Yukon, seul le diagramme d'Antifreeze Pond (RAMPTON, 1971) à $180 \mathrm{~km}$ à l'ouest d'Aishihik, livre la chronologie des étapes d'une afforestation régionale fondée sur l'augmentation des fréquences relatives de Picea: apparition de la forêt ouverte d'épinettes (15 à 20\% de grains de pollen de Picea) vers 8710 BP (GSC 1242) et fermeture de la forêt (25 à $30 \%$ de grains de pollen de Picea) vers 5690 BP (GSC 1040). À environ $18 \mathrm{~km}$ au NE du site du pingo, dans la vallée du ruisseau Mackintosh, un échantillon de gyttja daté de $7600 \pm 100$ BP (GSC 3952) montre qu'on n'a pas encore atteint la fermeture de la forêt puisque Picea ne représente que $15 \%$ du spectre pollinique.

Par conséquent, comparativement aux sites d'Antifreeze Pond et du ruisseau Mackintosh, les sédiments du flanc $N$ du pingo se sont déposés avant et pendant la phase d'afforestation régionale. Une question demeure néanmoins: l'aggradation du pergélisol a-t-elle été contemporaine de la sédimentation? 
Si on considère que la tranche d'eau diminue d'épaisseur par l'extension des formations palustres dans les mares résiduelles et les anciens chenaux (augmentation des valeurs cumulées des Cypéracées et d'Equisetum au centre du diagramme pollinique), dans ce cas le plancher peut être atteint par le gel hivernal. Si le gel est suffisamment profond, le plancher ne dégèle pas entièrement pendant l'été. C'est ce qui a été observé lors des sondages thermiques, le 11 août 1984 , dans le marais qui entoure le pingo.

Le diagramme pollinique montre aussi deux diminutions nettes des fréquences relatives des Cypéracées et d'Equisetum, l'une à $55 \mathrm{~cm}$ et l'autre à $7 \mathrm{~cm}$. Ces réductions des espèces hydrophiles peuvent indiquer des phases de meilleur drainage strictement local et pourraient donc suggérer deux étapes de soulèvement du pingo. La première étape serait antérieure à l'époque du saupoudrage de la White River Ash et postérieure à la fermeture régionale de la forêt (dans le secteur d'Aishihik). L'aggradation du pergélisol aurait soulevé la partie centrale du pingo et mis en place la glace observée entre 100 et $115 \mathrm{~cm}$ de profondeur dans le site du sondage latéral sans toutefois le soulever suffisamment pour exonder ce site. En effet, on trouve des valeurs plus élevées de Cypéracées et d'Equisetum entre 50 et $12 \mathrm{~cm}$. La sédimentation s'est donc poursuivie malgré le pergélisol dans un site palustre. L'aggradation du pergélisol se serait poursuivie, aboutissant à un deuxième soulèvement après le dépôt de la White River Ash. Celui-ci aurait provoqué l'émergence du site du sondage latéral. II pourrait également être à l'origine de la rupture sommitale du pingo et des failles observées.

\section{CONCLUSION}

L'approche originale de cette étude, par sa pluridisciplinarité, a permis de rassembler les caractères morphogénétiques et le cadre temporel de la formation de la butte d'Aishihik.

Des considérations basées sur la forme et l'environnement avaient permis à HUGHES (1969) de proposer qu'il s'agisse d'un pingo de système fermé, mais sans plus de précision. L'ensemble des analyses effectuées, non seulement confirment I'hypothèse de Hughes, mais illustrent concrètement le modèle théorique de processus de croissance centrale proposé par MACKAY (1979), à savoir la convexité des lentilles de glace pure et les cassures.

En l'absence de datations au radiocarbone, la combinaison des indices fournis par la téphrochronologie, la palynologie et la stratigraphie régionale nous permettent de situer le soulèvement du pingo après la fermeture régionale de la forêt ( $\pm 5700 \mathrm{BP}$ ) et sa réactivation après le saupoudrage de la White Rive Ash (1230 BP).

L'intérêt particulier de ce pingo vient du fait qu'il s'est formé par pression hydrostatique, dans un système fermé, alors qu'il se situe à la limite sud du pergélisol discontinu. Ceci nous incite à minimiser l'importance des seuils thermiques proposés par divers auteurs (WASHBURN, 1979) pour mettre l'accent sur les paramètres environnementaux. Ceux-ci, par leur variation spatiale, peuvent localement présenter une combinaison "oro-hydrographique" favorable au développement d'un pingo.

\section{REMERCIEMENTS}

Cette recherche a été financée par le Conseil de recherche en sciences et génie du Canada, subventions A6888 et N0068. La sonde à pergélisol nous a été prêtée par la Commission géologique du Canada.

Nous exprimons toute notre gratitude au professeur Guy Seret de l'Université catholique de Louvain pour sa contribution à ce travail par ses analyses minéralogiques et ses nombreux commentaires critiques. Nous remercions aussi particulièrement Xiacheng Wang pour les analyses polliniques, lan Campbell et Guy Auger pour les dessins, ainsi que Hector Beaudet, Michel Desjardins et André Labossière pour leur aide sur le terrain.

Messieurs A. Pissart et J. Gray, ainsi que le comité de rédaction, ont fourni des suggestions qui ont permis d'améliorer le manuscrit.

\section{RÉFÉRENCES}

DENTON, G. H. et KARLÉN, W. (1977): Holocene glacial and treeline variations in White River valley and Skolai Pass, Alaska and Yukon Territory, Quaternary Research, vol. 7, p. 63-111.

HUGHES, O. L. (1969): Distribution of open-system pingos in Central Yukon Territory with respect to glacial limits, Geological Survey of Canada, Paper 69-34, 8 p.

LERBEKMO, J. F. et CAMPBELL, F. A. (1969) : Distribution, composition, and source of the White River Ash, Yukon Territory, Canadian Journal of Earth Sciences, 6, p. 109-116.

MACKAY, J. R. (1979): Pingos of the Tuktoyaktuk Peninsula Area, North-West Territories, Géographie physique et Quaternaire, vol. 33, p. 3-61.

PISSART, A. (1983): Pingos et Palses: un essai de synthèse des connaissances actuelles. Mesoformen des Reliefs im heutigen Periglazialraum, Abhandlungen der Akademie der Wissenschaften in Göttingen. Mathematish-Physikalische Klasse, Dritte Folge Nor. 35, p. 48-69.

POLLARD, W. H. et FRENCH, H. M. (1983) : Seasonal frost mound occurrence, North Fork Pass, Ogilvie Mountains, Northern Yukon, Canada, in Permafrost: Fourth International Conference, Proceedings, National Academy Press, Washington DC. p. 10001004.

RAMPTON, V. N. (1971): Late Quaternary vegetational and climatic history of the Snag-Klutlan area, southern Yukon Territory, Canada, Geological Society of America Bulletin, vol. 82, p. 959-978.

RITCHIE, J. C., CWYNAR, L. C. et SPEAR, R. W. (1983): Evidence from Northwest Canada for an early Holocene Milankovitch thermal maximum, Nature, vol. 305, p. 126-128.

SERVICE DE L'ENVIRONNEMENT ATMOSPHÉRIQUE (1982): Normales climatiques au Canada. Températures et précipitations. 1951-1980. Le Nord-T.Y. et T.N.-O., Environnement Canada, Ottawa, Centre d'édition du Gouvernement du Canada, $55 \mathrm{p}$.

VEILLETTE, J. J. et NIXON, F. M. (1980): Portable drilling equipment for shallow permafrost sampling, Geological Survey of Canada, Paper 79-21, 35 p.

WASHBURN, A. L. (1979): Geocryology. Arnold, New York, 406 p. - (1983): What is a palsa? Mesoformen des Reliefs im heutigen Periglazialraum, Abhandlungen der Akademie der Wissenschaften in Göttingen. Mathematisch-Physikalische Klasse, Dritte Folge Nor. 35 , p. 34-47. 